Rechtsmedizin 2021 · 31:62-66

https://doi.org/10.1007/s00194-020-00422-z

Online publiziert: 7 . September 2020

(c) Der/die Autor(en) 2020

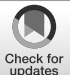

\author{
Marcus Schwarz' - Benjamin Ondruschka' · Carsten Babian' - Uwe Ebert ${ }^{2}$. \\ Jan Dreßler ${ }^{1}$ \\ 'Institut für Rechtsmedizin, Medizinische Fakultät, Universität Leipzig, Leipzig, Deutschland \\ ${ }^{2}$ Abteilung 6 Fachbereich 63 - Schusswaffen, Zweigstelle Leipzig, Landeskriminalamt Sachsen, Leipzig, \\ Deutschland
}

\title{
Jäger tot. Rehbock tot
} Kasuistik eines Jagdunfalls

\section{Einleitung}

Tödlich verlaufende Jagdunfälle sind selten. Gesonderte Statistiken zu diesem Thema werden nicht geführt. Erfragt man die Daten beim Deutschen Jagdverband (DJV), so liegt die Zahl der bei einer Jagd tödlich verunfallten Personen in den letzten 10 Jahren jährlich zwischen einer und 8 Personen. Hierbei werden sowohl Verstorbene gezählt, die durch Schusswaffen, aber auch andere Unfälle verstorben sind. Kommt es aber zu einem solchen Unfall, sind Rechtsmedizin und Kriminalpolizei regelmäßig in die Fallbearbeitung eingebunden, um ein Fremdverschulden auszuschließen.

Gerade bei Gesellschaftsjagden zur Erntezeit und auf Drückjagden kommt es aufgrund der höheren Teilnehmerzahl, der teilweise fehlenden Jagdpraxis der Teilnehmer und mancher ungewohnten Situation (beispielsweise Erntejagden mit schnell flüchtendem Wild), gelegentlich zu Personenschäden. Differenzialdiagnostisch kommen Verletzungen durch das bejagte Wild selbst in Betracht, wenn entgegen der gebotenen Vorsicht angeschossenem Wild nachgegangen wird und dieses den Jäger attackiert $[1,2]$. In der Mehrzahl dieser Fälle liegt ursächlich eine Nichteinhaltung von Sicherheitsbzw. Unfallverhütungsvorschriften oder mangelnde Grundfähigkeiten in der Jagd vor. Für über ein Drittel der tödlichen Jagdunfälle ist ein unsachgemäßer Umgang mit der Waffe verantwortlich [3]. Ungewöhnlich ist folgender Fall eines verstorbenen Jägers, welcher leblos neben einem ebenfalls toten Rehbock aufgefunden worden war.

\section{Kasuistik}

\section{Ereignisort}

Mitte Mai fuhr ein erfahrener Jäger, der über 25 Jahre im Besitz eines gültigen Jagdscheins war, gegen 19:00 Uhr in sein Jagdrevier. Als er zur vereinbarten Zeit am nächsten Morgen nicht wieder zu Hause eingetroffen war, habe sich sein Sohn auf die Suche nach ihm begeben und ihn leblos in Rückenlage mit seiner Jagdkleidung bekleidet auf einem Feld liegend vorgefunden. Dieser rief umgehend die Polizei, welche einen Notarzt hinzurief. Dieser konnte nur noch den Tod des Jägers feststellen; eine Reanimation wurde nicht mehr durchgeführt. Anschließend wurden Kriminalpolizei und Rechtsmedizin involviert.

Nach Zeugenangaben seien am Vortag gegen 19:50 Uhr 2 Schüsse aus der Richtung des späteren Leichenfundortes gehört worden.

Die rechtsmedizinische Leichenschau fand direkt am Fundort statt. Die Leiche wies spärliche Totenflecke entsprechend einer Rückenlage auf. Am Bauch und an der linken Leiste wurden perforierende Weichteilverletzungen festgestellt. Unter dem Toten fand sich eine größere Blutlache. Etwa $2 \mathrm{~m}$ entfernt lag ein toter Rehbock. Zwischen dem Rehbockkadaver und der Leiche des Mannes fand sich eine stark beschädigte Bockbüchsflinte der Marke Merkel (Merkel Jagd- und Sportwaffen, Suhl, Thüringen, Deutschland) (• Abb. 1).

Von der Kriminalpolizei wurde daraufhin die Hypothese aufgestellt, dass die Person womöglich vom Rehbock tödlich verletzt worden sein könnte. Das Tier wies eine Schussverletzung im oberen Viertel des rechten Schulterblatts (sog. Hochblattschuss) auf. Weitere äußerlich erkennbare Verletzungen des Tiers fanden sich bei der äußeren Untersuchung nicht. Eine Sektion des Rehbockkadavers wurde nicht beauftragt.

Aufgeworfene Erdspuren im Acker vor dem Rehbock wiesen darauf hin, dass der auf das Tier abgegebene Schuss nicht unmittelbar tödlich verlaufen war. Im Ergebnis der Leichenschau vor Ort war von einem Verblutungstod des Jägers auszugehen. Die Morphologie seiner Weichteilläsionen sprach für erlittene Schussverletzungen.

\section{Obduktionsbefunde}

Eine schräg-ovale Hautaufreißung mit zentralem Gewebedefekt sowie bläulichviolettem Unterblutungssaum fand sich am Unterbauch links. Ein Schusskanal durch das Unterhautfettgewebe des Bauches mündete schräg unterhalb inguinal davon in einer grobfetzigen, wundrandadaptierbaren Hautdurchtrennung als Ausschuss. Es lag damit ein tangentialer Durchschuss einer Bauchdeckenfalte ohne Eröffnung des Peritoneums vor. In einer dem Schusskanal folgenden, gedacht geradlinigen Fortsetzung bestand unterhalb des linken Leistenbandes eine schräg gestellte, breit klaffende Hautzerreißung (• Abb. 2). Der kräftig schwärzlich eingeblutete Schusskanal setzte sich von hier leicht nach dorsal ansteigend fort und verletzte dabei neben der Oberschenkelmuskulatur die Vasa femoralia und den Femurschaft bis zur Endlage der Projektilfragmente in den Gesäßweichteilen unterhalb der 


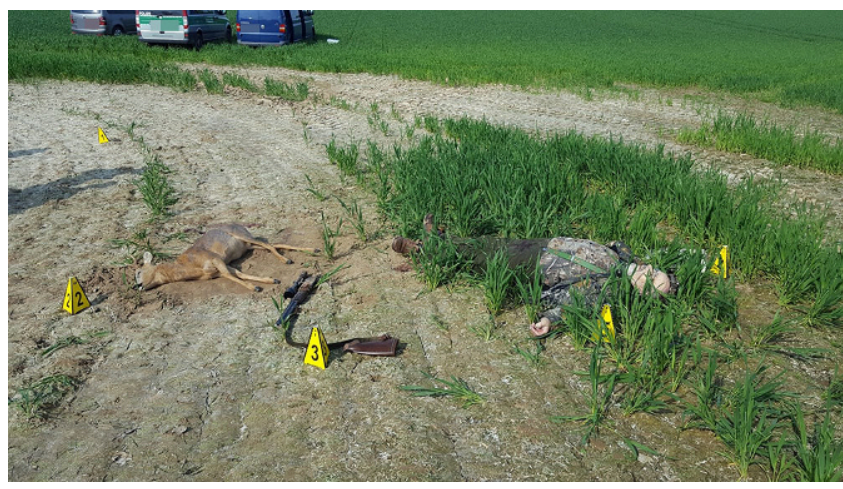

Abb. $1 \Delta$ Auffindesituation am Ereignisort

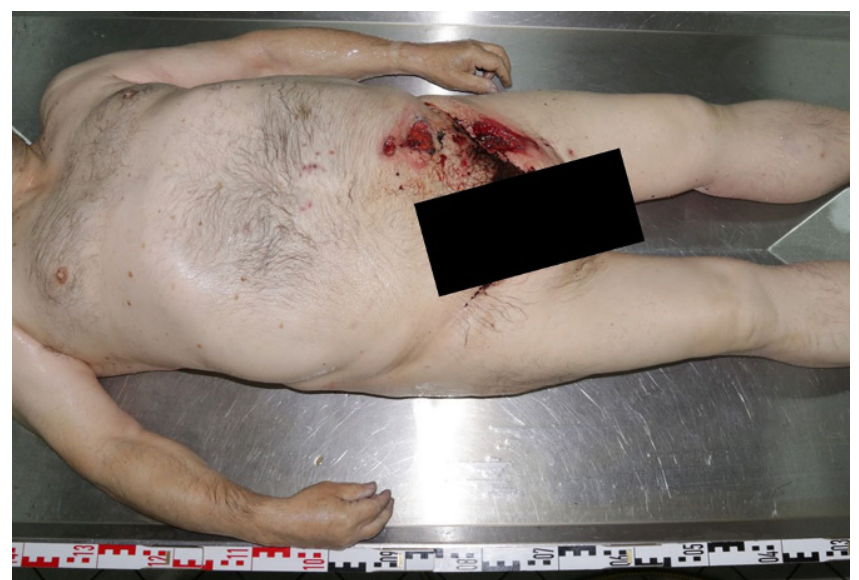

Abb. $2 \Delta$ Verletzungsbefunde vor der Obduktion (Illustration der Schusskanalverläufe: • Abb. 4)
Gesäßhaut. Durch den Schusskontakt mit dem Oberschenkelknochen war es zur Zerlegung des Projektils in mehrere Teile gekommen.

Durch spärliche Totenflecke, Blässe der inneren Organe und Schleimhäute, subendokardiale Blutungen des linksventrikulären Myokards und eine entspeicherte Milz mit gerunzelter Kapsel wurde der Tod durch Verbluten autoptisch bestätigt. Im Übrigen bestanden keine Organerkrankungen von todesursächlicher Bedeutung oder weitere Formen von Gewalteinwirkungen gegen den Körper. Es ergaben sich keine Hinweise auf eine stattgehabte notfallmedizinische oder laienhafte Versorgung.

Im Bauchbereich oberhalb des Nabels bestanden weiterhin frischere oberflächliche Hautabschürfungen (• Abb. 2) ohne korrespondierende Beschädigungen der Oberbekleidung (Hemd aus Baumwolle). Im Ergebnis der Obduktion waren diese nicht schussassoziiert entstanden.

Korrespondierend zu den Verletzungen am Leichnam bestanden Beschädigungen der Hose, welche mittels Hosenträgern vergleichsweise weit oben (Bund etwa auf Höhe des Bauchnabels) gehalten waren. Am primären Ein- und Ausschusses im Bauchbereich lagen einzeln voneinander abgrenzbare Beschädigungen vor; der sekundäre Einschuss der Leistengegend im Verlauf des Schusskanals in den Oberschenkel zeigte eine größere Textilbeschädigung, mit linksseitig kleinen, halbradiär gezeichneten Zerreißungen. Am Einschuss fanden sich schwärzliche Auflagerungen als charakteristische Hinweise auf Schmauch- und Pulverrückstände.

Im Rahmen der toxikologisch-chemischen Analysen wurden in den gängigen Untersuchungsmaterialien des Leichnams weder Alkohol, Drogen noch Medikamente nachgewiesen.

\section{Begutachtung von Waffe und Munition}

Die Waffe nebst Munition (• Abb. 3) sowie die asservierten Munitionsfragmente wurden zur ballistischen Charakterisierung gemeinsam mit dem LKA Sachsen untersucht. Es handelte sich um eine Bockbüchsflinte der Fa. Merkel mit einem Kugellauf im Kaliber $8 \times 57$ JRS und einem Schrotlauf im Kaliber 12/70. In den Patronenlagern steckten 2 abgefeuerte Patronenhülsen.

Die Waffe wies bei der Begutachtung mehrere Mängel auf: Das Abzugsgewicht lag bei $400 \mathrm{~g}$ (Norm: $>600 \mathrm{~g}$ ), was häufiger zum „Doppeln“ (gleichzeitiges Auslösen mehrerer Patronenlager) der Läufe führte und Kugel- und Flintenlauf gleichzeitig abschoss. Die automatische Abzugssicherung war verschlissen und funktionierte nicht einwandfrei. Dies führte $\mathrm{zu}$ einer automatischen Schussabgabe des Kugellaufs, wenn nur der Abzug des Flintenlaufs betätigt worden war und vice versa. Das Stechersystem (hier: sog. französischer Rückstecher) war in seiner Funktion gestört und ein Einstechen der Waffe damit nicht mehr möglich. Diese eigent- lich zur Minderung des Abzugswiderstandes direkt vor dem Schuss genutzte Waffenfunktion führte dazu, dass ein minimaler Druck am Abzug einen Schuss auslösen konnte. Die Waffe war hinter dem Abzug im Bereich des Griffstücks vollständig in zwei getrennt vorliegende Teile gebrochen.

Im Ergebnis dieser Waffenanalyse war festzuhalten, dass die Bockbüchsflinte auch ohne den frischen Materialbruch stark reparaturbedürftig und somit für einen sicheren jagdlichen Einsatz ungeeignet gewesen war.

Zum Materialbruch ist anzumerken, dass der Ursprung des Bruches an einer ca. $1,5 \mathrm{~cm}$ langen, in den Schaft gebrachten Schraube lokalisiert ist, welche den Abzugsbügel am Schaft der Waffe fixierte. Der Bruch verläuft weitestgehend glatt bis zur Oberseite des Schaftes, wo ein metallisches Zierteil der Waffensicherung den Bruch limitiert. Es ist anzunehmen, dass hier eine geschwächte Konstruktion („Schwachstelle") vorlag. Diese Überlegung wird weiterführend begründet durch die Annahme, dass das angenommene versuchte Erschlagen eines Tieres bereits in der Vergangenheit wiederholt ausgeführt worden war.

In der Waffe fanden sich im Kugellauf eine messingfarbene Patronenhülse mit einer Hülsenbodenprägung „RWS $8 \times 57$ JRS“ sowie im Flintenlauf eine Patronenhülse aus Kunststoff mit einer Hülsenbodenprägung „12* $12^{\star} 12^{\star} 12^{* \star}$. Beide Hülsen wiesen frische Verfeuerungsspuren auf. 
Aufgrund der in den Asservaten des Verstorbenen mitgeführten Munition konnte eine Delaborierung von Beispielprojektilen durchgeführt werden. Bei den Geschossen, die den Patronenhülsen mit der Hülsenbodenprägung „RWS $8 \times 57$ JRS“ zuzuordnen waren, handelte es sich um Torpedo-Ideal-Geschosse (TIG), welche über die Fa. Brenneke vertrieben werden und ein Geschossgewicht von $12,8 \mathrm{~g}$ aufwiesen. Es handelt sich hierbei um ein Zweikernteilzerlegungsgeschoss aus einem weichen, vorderen und einem härteren, hinteren Bleikern in einem nickelplattierten Stahlmantel. Der ballistische Vergleich zu den im Leichnam asservierten Geschossfragmenten zeigte hierzu eine vollständige Übereinstimmung. Die Patronenhülse im Flintenlauf wurde einem Rubin-SabotBrenneke-Geschoss im Kaliber 12/70 zugeordnet, welches ebenfalls über die Firma Brenneke (Brenneke Ammunition $\mathrm{GmbH}$, Langenhagen, Niedersachsen, Deutschland) vertrieben wird.

\section{Vorfallsrekonstruktion}

In Zusammenschau der Feststellungen am Fundort sowie der Ergebnisse der rechtsmedizinischen Sektion und der waffentechnischen Untersuchungen wurden mit einer bauartgleichen Waffe verschiedene Situationen zum Moment der (unfreiwilligen) Schussabgabe nachgestellt. Dabei wurden die Neigung/ Beugung des Oberkörpers nach vorn bzw. das Schrittmaß bei einer möglichen Ausholbewegung variiert. Abschließend kam bei den Versuchen nur eine Körperhaltung plausibel in Betracht, da ansonsten andere Schusskanalverläufe erzeugt worden wären: Beim initialen Schuss auf den Rehbock war der Flintenlauf abgefeuert worden. Dieses RubinSabot-Brenneke-Geschoss im Kaliber 12/70 traf den Rehbock dabei nicht an der gewünschten Stelle (Blattschuss) und führte damit nicht zum sofortigen Tod. Der Jäger hat sich daraufhin mit noch geladener Waffe (projektilgeladener Kugellauf) auf den Weg zum liegenden Rehbock begeben. Unter Berücksichtigung des Schaftbruchs der Waffe ist anzunehmen, dass diese als keulenähnlicher Schlaggegenstand Verwendung finden sollte. Dabei muss die Waffe am Laufpaar gegriffen worden sein, um den Kolben der Bockbüchsflinte als Schlagstück zu verwenden. Durch die dabei anzunehmende leicht nach vorn gebeugte Körperhaltung bei der Schlagbewegung ist der autoptisch dargestellte Schusskanalverlauf plausibel erklärbar. Die Projektilfragmente fanden nach Zerlegung am Oberschenkelknochen des Jägers ihre Endlage im Gesäß. Der Schusskanalverlauf der Fragmente steigt dabei bogenförmig nach oben an, was in - Abb. 4 zwischen den Punkten 3 und 4 illustriert wurde. Das Verletzungsbild am Jäger spricht dafür, dass die (unbeabsichtigte) Schussabgabe bereits während einer Bewegung zum Ausholen bzw. Niederschlagen stattgefunden haben muss und nicht - wie zwischenzeitlich vermutet - beim Auftreffen der Waffe auf dem Boden oder dem Rehbock (visualisiert in - Abb.4). Zum Bruch der Waffe kam es, als diese nach der, durch den Schuss unterbrochenen schwungvollen Schlagbewegung, zu Boden fiel. Dafür spricht auch, dass am Kadaver des Rehbocks keine äußerlich erkennbaren Folgen einer stumpf-kantigen Gewalteinwirkung festzustellen waren, obgleich solche aus rechtsmedizinischer Erfahrung selbst nach einem Treffer nicht zwingend äußerlich hätten erkennbar sein müssen.

Polizeiliche Umfeldbefragungen ergaben, dass vom nunmehr Verstorbenen die Praxis des Erschlagens der getroffenen Tiere bereits in der Vergangenheit angewendet worden wäre: „Das hat er schon immer so gemacht."

In der „Unfallverhütungsvorschrift Jagd“ in der Fassung vom 01.05.2017 [4] ist in $\$ 2$ Waffen und Munition Absatz 1 notiert, dass nur Schusswaffen verwendet werden dürfen, die „den Bestimmungen des Waffengesetzes entsprechen und nach dem Bundesjagdgesetz für jagdliche Zwecke zugelassen sind. Die Waffen müssen funktionssicher sein und dürfen nur bestimmungsgemäß verwendet werden“. Ergänzend findet sich in der Durchführungsanweisung zu Absatz 1 im Punkt II weiter: „Keine bestimmungsgemäße Verwendung ist z. B. die Benutzung der Waffe zum ... Erschlagen des Wildes“.
Rechtsmedizin 2021 · 31:62-66

https://doi.org/10.1007/s00194-020-00422-z

(c) Der/die Autor(en) 2020

M. Schwarz - B. Ondruschka - C. Babian . U. Ebert · J. Dreßler

\section{Jäger tot. Rehbock tot. Kasuistik eines Jagdunfalls}

\section{Zusammenfassung}

Ein erfahrener Jäger kehrte nach einem Abendansitz auf Rehwild nicht nach Hause zurück. Er wurde am folgenden Morgen von seinem Sohn auf einem Feld, in einer Blutlache liegend, tot aufgefunden. Neben inm lag ein toter Rehbock. Der Jäger wies eine stark blutende Bauchverletzung auf, welche die Polizei zur Spekulation veranlasste, der Rehbock hätte diese Verletzung mit seinem Geweih verursacht. Zudem lag zwischen dem Jäger und dem Kadaver ein Gewehr mit zerbrochenem Schaft. Im vorgestellten Fall wurden mehrere sicherheitsrelevante Aspekte im Umgang mit Schusswaffen und Grundregeln der Jagd ignoriert, was zu einem tödlichen Jagdunfall führte.

Schlüsselwörter

Wundballistik - Unfall · Schusswaffen . Rekonstruktion · Verbluten

\section{Hunter dead. Roebuck dead. Case report of a hunting} accident

\section{Abstract}

An experienced hunter did not return home after an evening sitting for roe deer. He was found dead the following morning by his son in a field, lying in a pool of blood. Next to him lay a dead roebuck. The hunter had a heavily bleeding abdominal injury, which led the police to speculate that the roebuck had caused this injury with his antlers. There was also a rifle with a broken stock between the hunter and the deer carcass. In the case presented, several safety-relevant aspects of the handling of firearms and basic rules of hunting were ignored and led to a fatal hunting accident.

\section{Keywords}

Wound ballistics - Accident - Guns .

Reconstruction - Exsanguination 


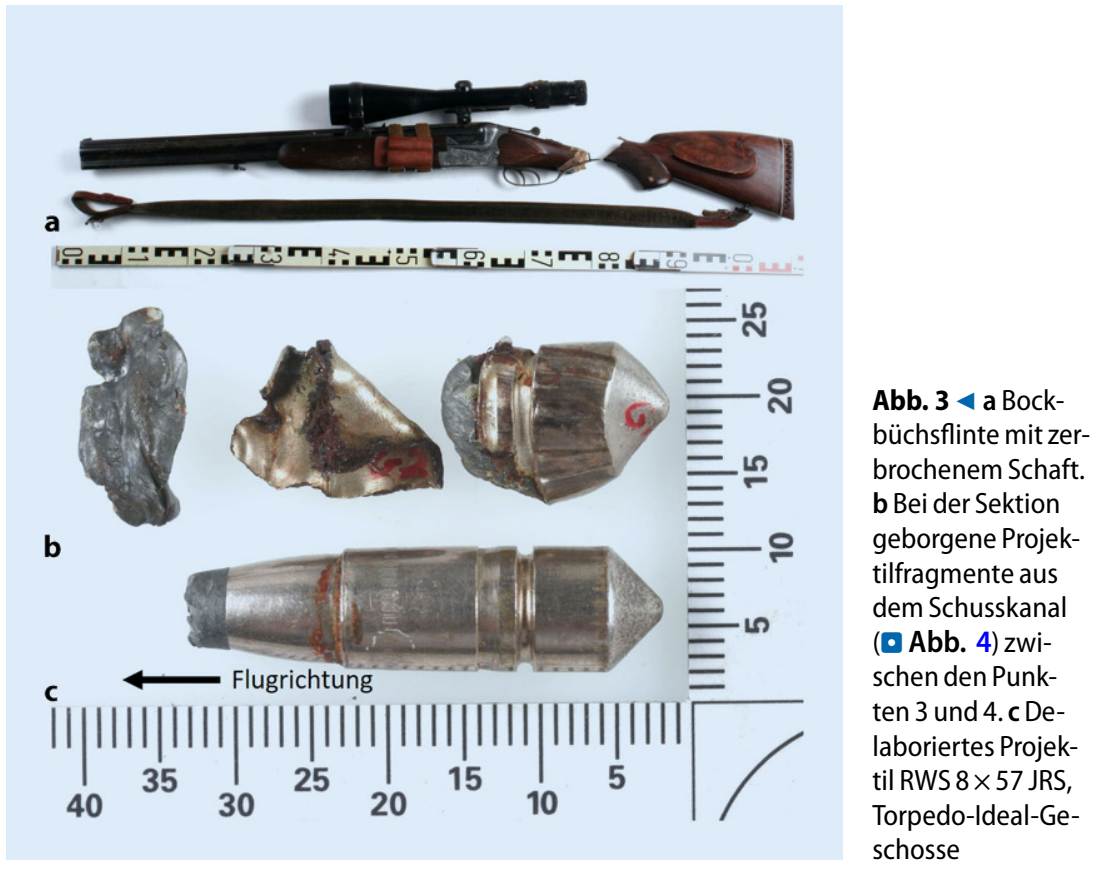

\section{Diskussion}

Sterbefälle während einer Jagd sind selten und bergen oft Besonderheiten. Ermittlungsbehörden sind daher angehalten, durch die Anordnung rechtsmedizinischer Untersuchungen zu klären, ob ein Fremdverschulden in Betracht zu ziehen ist oder ein Unfallgeschehen vorliegen könnte. Die gemeinsame Fallbearbeitung durch Kriminalpolizei, Rechtsmedizin und Waffensachverständigen hat im vorliegenden Fall den Hergang aufklären und einen Straftatverdacht ausräumen können: Die Verkettung von Missachtungen mehrerer Gesetze, Empfehlungen, Unfallverhütungsvorschriften und jagdlich/waidmännischer Bräuche sowie der Verwendung einer verschlissenen Jagdwaffe führte zu einem tödlich endenden Jagdunfall. Aus einer nicht einwandfrei funktionierenden, geladenen und nichtgesicherten Waffe löste sich bei unsachgemäßer Handhabung als Schlagwerkzeug ein Schuss (• Abb. 4).

Wünschenswert wäre die zusätzliche Sektion des toten Rehbocks gewesen, welche aber von der Staatsanwaltschaft nicht beauftragt worden war.

In einer Auswertung von Schussverletzungen in einem Ballungszentrum wurden tödlich endende Verletzungen der Extremitäten nur selten beobachtet [5], wie bekannt sind tödliche Unfälle durch Schusswaffen per se Raritäten [6]. Demgegenüber beziffert sich der Anteil an Extremitätenverletzungen bei schussbedingten Jagdunfällen auf ca. ein Viertel dieser Fälle [3], wie im vorliegenden Fall dargestellt. Neben Jagdunfällen werden gelegentlich auch unfallbedingte Sterbefälle vom Sportschießen referiert [7].

Insbesondere die aus Langwaffen verschossenen Munitionen bieten ein hohes Verletzungspotenzial [8]. Der Wunsch nach hohem Wirkungsgrad der Geschosse zur schnellen Tötungswirkung wird für die Jagd durch Verwendung von Teilmantelgeschossen mit hohem Deformationspotenzial erfüllt. Die hier verwendete Munition ist bereits seit über 100 Jahren speziell für diesen Zweck auf dem Markt erhältlich. Durch die unterschiedlich harten Bleikerne des Geschosses wird die Projektilwirkung dabei noch verstärkt. Durch die zunehmenden Wundgrößen ist anzunehmen, dass sich das verschossene Projektil bereits beim ersten Auftreffen auf der Körperoberfläche am linken Unterbauch verformte hatte. Im Verlauf des Schusskanals zerlegte sich das Geschoss am Femurschaft und führte zum fatalen Verletzungsbild.

Im referierten Fall hätte aufgrund der Lokalisation der Verletzung in der Leistengegend und damit schlecht zugänglicher Lokalisation für eine Tourniquet-

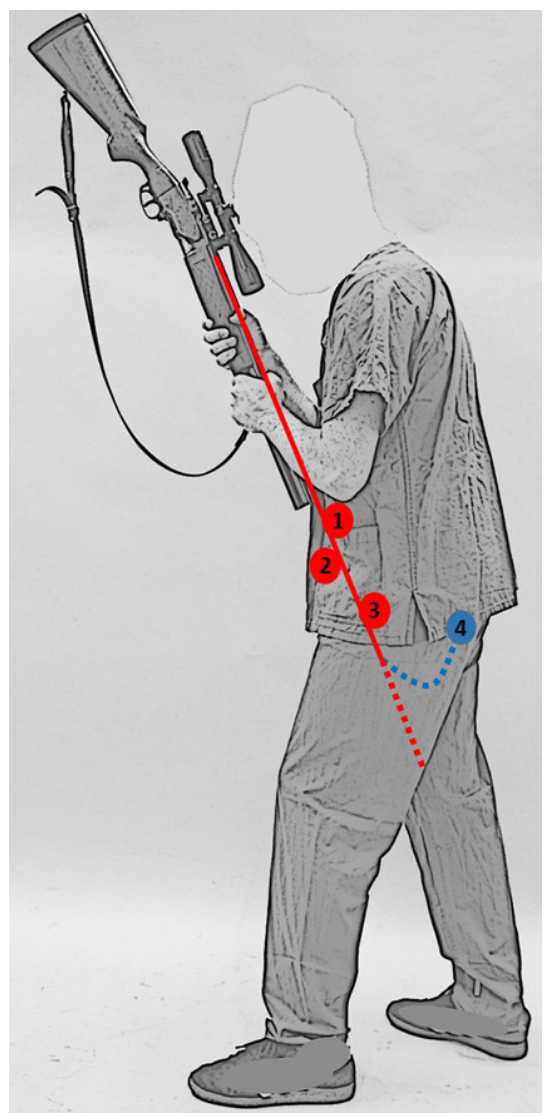

Abb. $4 \Delta$ Illustration der wahrscheinlichsten Körperhaltung bei Schussabgabe mit initialem Ein- und Ausschuss an der Bauchdeckenfalte $(1+2)$, sekundärem Einschuss inguinal (3; rot markiert) und Endlage der vom Oberschenkel abgelenkten Projektilfragmente glutäal (4; bogenförmig blau markiert) mit einer bauartgleichen Waffe

Versorgung auch bei sofortiger notfallmedizinischer Versorgung nach Erleiden der Verletzung nur eine geringe Überlebenschance bestanden $[9,10]$.

\section{Fazit für die Praxis}

- Zwischenfälle mit Schusswaffen ereignen sich bei der Jagd nur bei Missachtung von Sicherheitsvorschriften und einschlägigen Richtlinien.

- Tödliche Jagdunfälle bedürfen einer gemeinsamen Untersuchung durch Kriminalpolizei, Rechtsmedizin und Waffensachverständigen zur Hergangsrekonstruktion und zum Ausschluss einer Fremdeinwirkung. 


\section{Korrespondenzadresse}

\section{Marcus Schwarz, M.Sc.}

Institut für Rechtsmedizin, Medizinische Fakultät, Universität Leipzig

Johannisallee 28, 04103 Leipzig, Deutschland marcus.schwarz@medizin.uni-leipzig.de

Funding. Open Access funding provided by Projekt DEAL.

\section{Einhaltung ethischer Richtlinien}

Interessenkonflikt. M. Schwarz, B. Ondruschka, C. Babian, U. Ebert und J. Dreßler geben an, dass kein Interessenkonflikt besteht.

Für diesen Beitrag wurden von den Autoren keine Studien an Menschen oder Tieren durchgeführt. Die Untersuchungen erfolgten unter Einhaltung der Vorgaben der Zentralen Ethikkommission der Bundesärztekammer.

Open Access. Dieser Artikel wird unter der Creative Commons Namensnennung 4.0 International Lizenz veröffentlicht, welche die Nutzung, Vervielfältigung, Bearbeitung, Verbreitung und Wiedergabe in jeglichem Medium und Format erlaubt, sofern Sie den/die ursprünglichen Autor(en) und die Quelle ordnungsgemäß nennen, einen Link zur Creative Commons Lizenz beifügen und angeben, ob Änderungen vorgenommen wurden.

Die in diesem Artikel enthaltenen Bilder und sonstiges Drittmaterial unterliegen ebenfalls der genannten Creative Commons Lizenz, sofern sich aus der Abbildungslegende nichts anderes ergibt. Sofern das betreffende Material nicht unter der genannten Creative Commons Lizenz steht und die betreffende Handlung nicht nach gesetzlichen Vorschriften erlaubt ist, ist für die oben aufgeführten Weiterverwendungen des Materials die Einwilligung des jeweiligen Rechteinhabers einzuholen.

Weitere Details zur Lizenz entnehmen Sie bitte der Lizenzinformation auf http://creativecommons.org/ licenses/by/4.0/deed.de.

\section{Literatur}

1. Dokter M, Philipp KP, Bockholdt B (2018) Der vermeidbare(?) Jagdunfall - Tödliche Oberschenkelverletzung durch ein Wildschwein. Rechtsmedizin 28:360

2. Philipp KP (1985) Tödlicher Jagdunfall durch Schwarzwild. KriminalistikForens Wiss 57:121-123

3. Karger B, Wissmann F, Gerlach D, Brinkmann B (1996) Firearm fatalities and injuries from hunting accidents in Germany. Int J Legal Med 108:252-255

4. Sozialversicherung für Landwirtschaft, Forsten und Gartenbau (2000in) „Unfallverhütungsvorschrift Jagd" mit Stand 1. Januar 2000 in der Fassung vom 1. Mai 2017. https://cdn.svlfg.de/ fiona8-blobs/public/svlfgonpremiseproduction/ 4602f00372a5a47d/bdad00376879/vsg4_4-jagd. pdf.Zugegriffen: 26. Mai 2020
5. Buschmann CT, Fricke A, Tsokos M, Hartwig S (2015) Schusstodesfälle im Land Berlin von 2000 bis 2009. Rechtsmedizin 25:130-138

6. Desinan L, Mazzolo GM (2005) Gunshot fatalities: suicide, homicide or accident? A series of 48 cases. Forensic Scilnt 147:37-40

7. Guilbert S (2008) Violence and accidents in competition sports. Sport Soc Cult Commer Media Polit 11:17-31

8. Martille L, Symes SA (2019) Interpretation of long bones ballistic trauma. Forensic Sci Int 302:109890

9. Ondruschka B, Baier C, Dreßler J, Höch A Bernhard M, Kleber C, Buschmann C (2017) Additional emergency medical measures in trauma-associated cardiac arrest. Anästhesist 66:924-935

10. Hossfeld B, Lechner R, Josse F, Bernhard $M$, Walcher F, Helm M, Kulla M (2018) Prehospital application of tourniquets for life-threatening extremity hemorrhage: systematic review of literatutre.Unfallchirurg 121:516-529
In eigener Sache

Autoren

WERKSTATT

GRATIS

Ein Service für Autoren von Springer Medizin

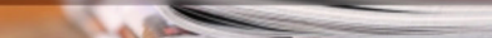

Fortbildungen für

Autor*innen und Gutachter*innen

Die ersten Veröffentlichungen sind für Jeden ein wichtiger Schritt in der angestrebten Karriere. Wissenschaftliche Artikel sind entscheidend dafür, dass die eigene Arbeit in der Community wahrgenommen wird. Es geht darum, die eigenen Ideen national und international auszutauschen und sicherzustellen, dass die Ergebnisse Wirkung erzielen.

Die Online-Kurse der Autorenwerkstatt helfen, sich leicht einen Überblick über das Schreiben, Einreichen, Begutachten und Veröffentlichen eines Manuskripts zu verschaffen.

5 Online-Kurse zu den wichtigsten Standards des wissenschaftlichen Publizierens:

- Wie verfasse ich ein Manuskript?

- Writing in English für deutschsprachige Autorinnen und Autoren

- Wie funktionieren Publikation und Begutachtung?

- Anleitung zur Open-AccessVeröffentlichung

- Leitfaden zur Peer-ReviewBegutachtung

Für alle, die auf SpringerMedizin.de registriert sind!

Jetzt gratis fortbilden unter www.springermedizin.de/ autorenwerkstatt/ 